\title{
Computed Tomographic Findings in 50 Cases of Gall Bladder Carcinoma
}

\author{
Lt Col RA George*, Col SC Godara ${ }^{+}$, Lt Col P Dhagat", Maj PP Som**
}

\begin{abstract}
Background : A retrospective assessment of contrast enhanced computed tomography (CECT) scan findings in histopathologically proven cases of carcinoma of the gallbladder (GB) was performed to review its role in diagnosis, staging and assessment of surgical resectability.

Methods : All the patients had been subjected to a standardised abdominal helical computed tomography scan. Orally administered iodinated contrast was used for opacification of bowel and dynamic intravenous injection of non-ionic iodinated contrast for studying the lesional enhancement and vascular structures.

Results : The presence of focal or diffuse mass lesions in the gallbladder fossa, infiltration of a liver and second part of duodenum were the most reliable diagnostic features in carcinoma gallbladder. Regional spread was better delineated on CT scan as compared with ultrasonography.

Conclusion : CT scan is an effective method for evaluating, characterizing and detecting the spread of GB carcinomas.
\end{abstract}

MJAFI 2007; 63 : 215-219

Key Words : Gall Bladder; Carcinoma; Computed Tomography

\section{Introduction}

$\mathrm{C}$ arcinoma of the gall bladder (GB) is the fifth most common malignancy of the gastrointestinal tract and the commonest malignancy of the biliary tree $[1,2]$. It occurs primarily in the sixth to seventh decade of life and is four to five times more common in women [2,3]. Ultrasonography (USG) and computed tomography (CT) have revolutionized the diagnosis and management of carcinoma GB. Magnetic Resonance Imaging (MRI) is utilized only in inoperable cases with obstructive jaundice for delineation of the biliary tract anatomy in patients considered for palliative stenting [3].

USG in patients of carcinoma GB has certain limitations such as interference by bowel gas, limited depth resolution and inadequate visualization of parts of the gallbladder in the region of posterior acoustic shadowing in the presence of calculi. CT scan overcomes these drawbacks and provides definite information regarding invasion of the tumour into the adjacent organs, distant metastasis, delineation of the biliary tree and portal vein involvement. We present our experience of CT findings in 50 histologically proven cases of carcinoma GB.

\section{Material and Methods}

This retrospective study reviewed CT examination findings in 50 histopathologically proven (from operative specimen or fine needle aspiration cytology) cases of carcinoma GB over a period of two years. The patients presented with a wide spectrum of complaints ranging from dyspepsia, abdominal pain to clinically palpable lump abdomen and obstructive jaundice. All the patients were subjected to helical CT scan examination on a GE HiSpeed CT/i scanner.

The CT protocol of $5 \mathrm{~mm}$ helical CT sections in the axial plane at table speed of $5 \mathrm{~mm} / \mathrm{sec}$ (pitch 1.0) with $\mathrm{kV} 120$ and $\mathrm{mA} 280$ was used. Breath-hold periods varied from 10 to 16 seconds with intervening breathing time of 8 seconds. The CT scan technique employed in our patients included a preliminary survey scan of the abdomen from levels of the diaphragm to the iliac crest after orally administered one litre of iodinated contrast $(800 \mathrm{ml}$ administered 45 minutes prior to and $200 \mathrm{ml}$ given just prior to the scan) for opacification of bowel during customized breath-hold phase. This was followed by repeat evaluation of the abdomen during dynamic intravenous injection of $80 \mathrm{ml}$ of non-ionic iodinated contrast medium (300 $\mathrm{mg}$ Iodine $/ \mathrm{ml}$ ) for studying the lesional enhancement and vascular structures. Intravenous contrast medium was administered at the rate of $2 \mathrm{ml} / \mathrm{sec}$ using a pressure injector at $150 \mathrm{psi}$ using a scan delay of 30 seconds to commence imaging. Suspected cases of duodenal involvement were subjected to targeted area scanning with patient in right decubitus position.

Results

There was definite female preponderance with male: female

${ }^{*}$ Classified Specialist (Radiology), ${ }^{\#}$ Graded Specialist (Radiology), Command Hospital (EC), Kolkata. ${ }^{+}$Commanding Officer, AFMSD, Pune. ${ }^{* *}$ Graded Specialist (Radiodiagnosis), Military Hospital Establishment No. 22, C/o 56 APO.

Received : 12.01.2005; Accepted : 27.06.2006 
ratio of 2:5 with the gender ratio increasing from 1:2 below 50 years of age to 1:3 in the peak incidence age group of 51-70 years (Table 1). The age of patients ranged from 34 to 76 years with a mean age of 57 years for females and 52 years for males.

There were three patterns of findings seen in carcinoma gall bladder (Table 2). 28 (56\%) cases presented with a mass in the GB fossa (Fig. 1), with half of these patients presenting in Stage T4 of the TNM system. A localized/ polypoidal growth within the GB was appreciated in $10(20 \%)$ cases (Fig.2) .Wall thickening of varying degrees either focal or diffuse was detected in 12 (24\%) cases (Fig. 3).

Infiltration into liver was seen in $36(72 \%)$ cases, while infiltration into the adjacent structures such as duodenum, pancreas and omentum was seen in $23(46 \%)$ cases at the time of diagnosis (Fig. 4). The lymph nodes (Fig. 1) were involved in $21(42 \%)$ patients. The lymph nodes around the celiac axis and at the porta hepatis (N1 stage) were involved in six and retroperitoneal nodal involvement (N2 stage) was seen in the remaining 15 patients.

Obstructive jaundice with intrahepatic biliary radicle dilatation (Figs. 1,4) was seen in $15(30 \%)$ cases and the commonest site of obstruction was the level of confluence of the left and right main hepatic ducts. Cholelithiasis in 29 $(58 \%)$, ascites in seven and pulmonary metastasis was seen in one case. Portal vein invasion and hepatic lobar atrophy were not identified in any of the cases in this series.

Findings were stratified using the TNM system following the contrast enhanced CT study [3]. In the TNM system, T1 tumours involve only the mucosa and muscle layer, T2 tumours involve the peri-muscular connective tissue, T3

Table 1

Age and sex distribution

\begin{tabular}{lrrrc} 
Age Group(yrs) & \multicolumn{2}{c}{ Number of cases } & Total & Percentage \\
& F & M & & \\
$? 30$ & - & - & - & - \\
$31-40$ & 3 & 2 & 5 & 10 \\
$41-50$ & 8 & 4 & 12 & 24 \\
$51-60$ & 11 & 4 & 15 & 30 \\
$61-70$ & 12 & 3 & 15 & 30 \\
$? ? 0$ & 2 & 1 & 3 & 6 \\
Total & 36 & 14 & 50 & 100
\end{tabular}

Table 2

Distribution of pathology

\begin{tabular}{lccccc} 
Findings & \multicolumn{2}{c}{$\begin{array}{c}\text { TNM stage } \\
\text { at diagnosis }\end{array}$} & $\begin{array}{l}\text { Number } \\
\text { of cases }\end{array}$ & Percentage \\
& T1 & T2 T3 & T4 & & \\
\hline
\end{tabular}

$\begin{array}{lllllll}\text { Wall thickening } & 3 & 3 & 6 & - & 12 & 24\end{array}$

(focal/diffuse)

$\begin{array}{lrrrrrr}\text { Focal mass } & 1 & 4 & 5 & - & 10 & 20 \\ \text { Mass replacing } & - & 8 & 6 & 14 & 28 & 56\end{array}$

GB in the GB fossa

$\begin{array}{lllllll}\text { Total } & 4 & 15 & 17 & 14 & 50 & 100\end{array}$

tumours extend beyond the serosa but involve less than $2 \mathrm{~cm}$ of the liver and T4 tumours invade beyond $2 \mathrm{~cm}$ of the liver. $\mathrm{N} 1$ denotes nodes in the cystic duct and portal groups while $\mathrm{N} 2$ indicates involvement of retro-pancreatic, aorto-caval and superior mesenteric lymph node groups. The TNM system (Table 3) findings were used to stage the disease with stage I and II representing T1 and T2 tumours without nodal or distant metastases, T3 and/or N1 disease as stage III, T4 tumours with N1/N0 were included in stage IVA whereas T4 tumours with distant nodal involvement or metastases (N2; M1) were considered as stage IVB.

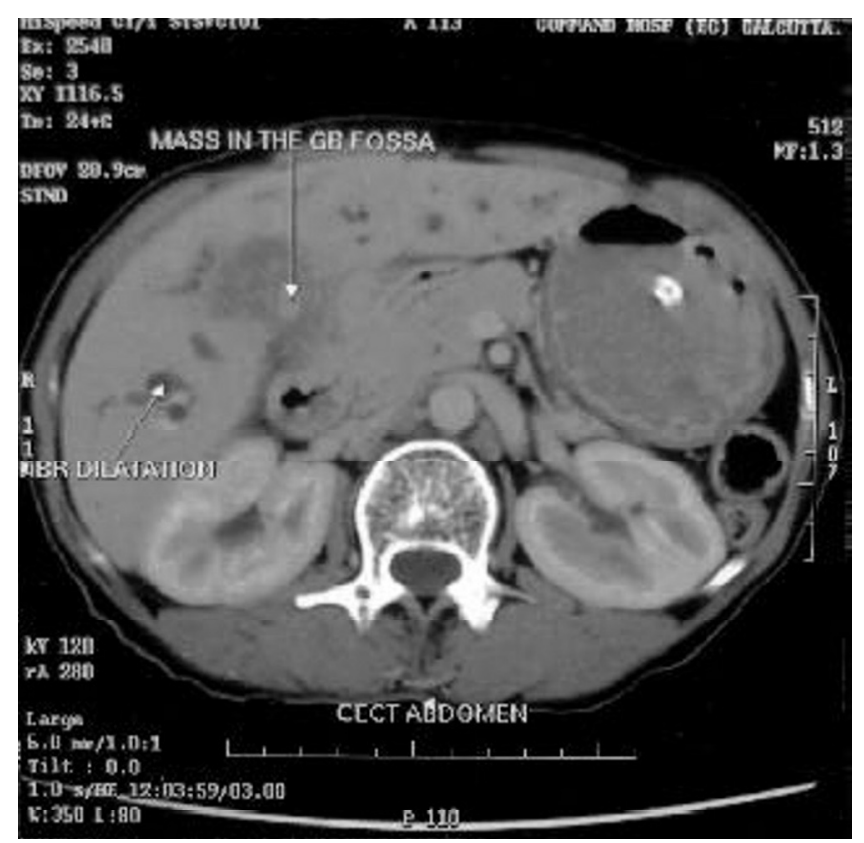

Fig. 1 : Axial contrast enhanced CT section of the abdomen showing mass replacing the GB with infiltration of the adjacent liver and stomach, with dilated intrahepatic biliary radicles

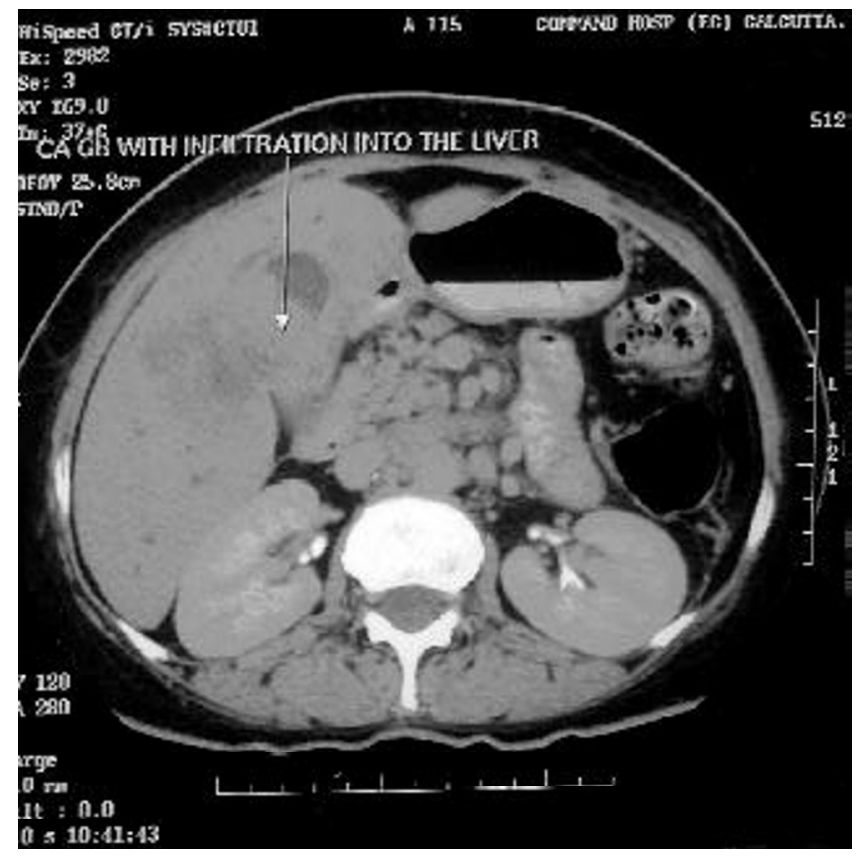

Fig. 2: Axial contrast enhanced CT section of the abdomen showing focal mass within the GB and multiple enlarged retroperitoneal and peri-pancreatic lymph nodes 


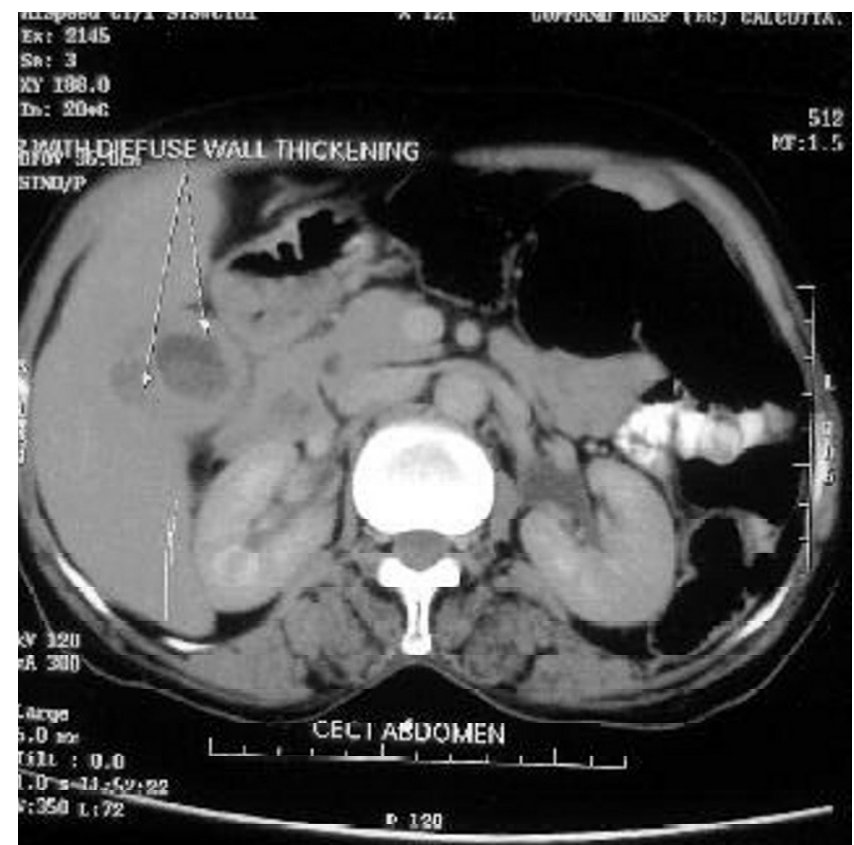

Fig. 3 : Axial contrast enhanced CT section of the abdomen showing diffuse concentric wall thickening of the GB

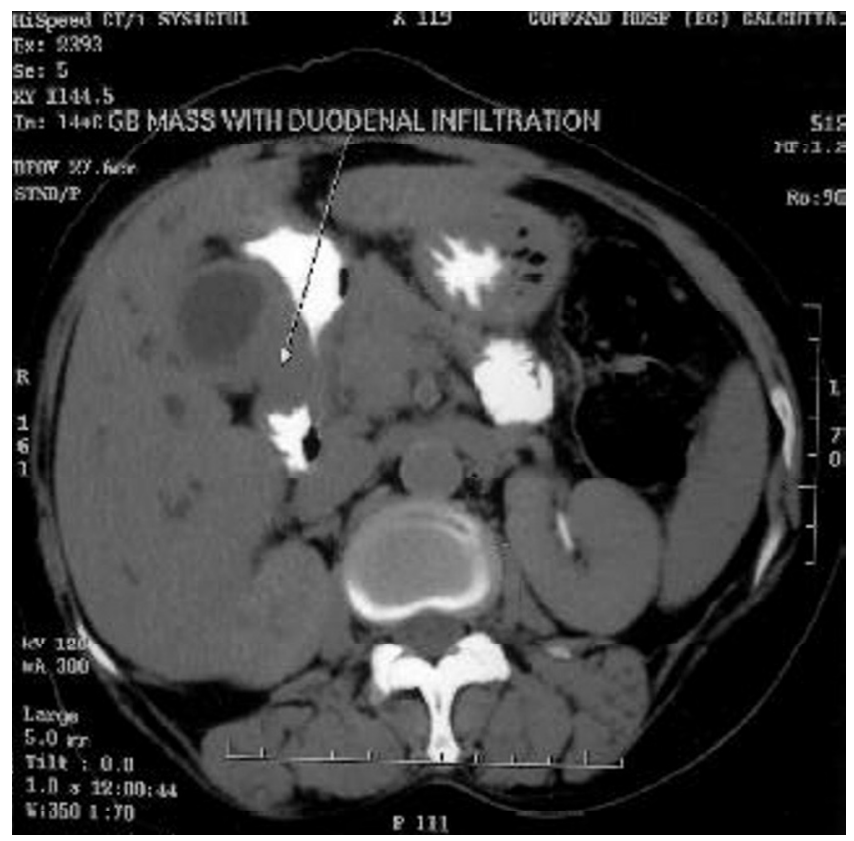

Fig. 4: Axial contrast enhanced CT section of the abdomen showing GB mass infiltrating the duodenum with dilated intrahepatic biliary radicles

The study findings are tabulated in Table 4. It was seen that patients having an imaging morphology of GB mass occupying the GB fossa formed the bulk of the cases, with disease stage at diagnosis being Stage IV in 29 (58\%) patients. It was also noted that as the disease progressed from Stage I to Stage IV there was a definite pattern of change in the morphologic pattern of the disease, with earliest stages being only focal or diffuse wall thickening, intermediate stage of focal mass and advanced stage of large mass replacing the entire gallbladder.
Table 3

CT staging : TNM and Modified Nevin system with staging protocol

$\begin{array}{lll}\text { Stage } & \text { TNM system } & \text { Modified Nevin system } \\ \text { I } & \text { T1N0M0 } & \text { In-situ carcinoma } \\ \text { II } & \text { T2N0M0 } & \text { Mucosal/ muscular invasion } \\ \text { III } & \text { T3N0M0 } & \text { Transmural direct liver invasion } \\ & \text { T3N1M0 } & \\ \text { IV A } & \text { T4N0M0 } & \text { Lymph nodal metastasis } \\ & \text { T4N1M0 } & \\ \text { IV B } & \text { TxN2M0 } & \text { Distant metastasis } \\ & \text { TxNxM1 } & \end{array}$

Table 4

CT staging: Number of cases and dominant morphologic types

$\begin{array}{lclr}\begin{array}{l}\text { Disease stage } \\ \text { at diagnosis }\end{array} & \text { No of cases } & \begin{array}{l}\text { Commonest morphologic type } \\ \text { (No of cases) }\end{array} \\ \text { Stage I } & 04 & \text { Wall thickening } \\ \text { Stage II } & 09 & \text { Focal mass } \\ \text { Stage III } & 08 & \text { GB mass/focal mass } & (5) \\ \text { Stage IV A } & 07 & \text { GB mass } \\ \text { Stage IV B } & 22 & \text { GB mass }\end{array}$

\section{Discussion}

Patients of GB carcinoma present with complaints ranging from dyspepsia, malaise, weight loss, jaundice to lump abdomen $[1,2]$. Those with obstructive jaundice usually present in advanced stages of the disease [3] and the five year survival in such cases is less than 5\% [4]. Majority of the malignancies of the GB are adenocarcinomas [5]. In this study 49 cases were confirmed as adenocarcinoma, while one case was diagnosed as squamous cell carcinoma. The exact aetiology of carcinoma GB is unknown, however, several associated risk factors have been identified. The closest association is with cholelithiasis, which is seen in $65-$ $95 \%$ of patients as reported in different series. Other factors related are calcification of GB wall (porcelain GB), genetic factors, anomalous pancreatic biliary duct junction, infections and environmental carcinogens [15], of which the association with cholelithiasis is the most established [2]. In our study, 29(58\%) patients had associated gallstones.

In the present study, the male : female ratio was $2: 5$ and the mean age of presentation was 57 years for females and 52 years for males, which is almost a decade less than the reported mean age in western literature. Similar observations have been reported earlier by Kumar et al [6], in their experience of 116 cases of carcinoma GB in North India. This variation may represent the regional disease pattern or indicates the changing trend in the disease statistics as a 
consequence of the improved imaging modalities.

One hypothesis of carcinoma GB genesis suggests that irritation of gallbladder mucosa by stones causes chronic inflammation, followed by repetitive epithelial repair, which may lead to malignant transformation. Approximately 15 years is required for dysplasia to progress to invasive carcinoma [5]. In this context, the change in morphologic pattern of the disease was noted as the disease progressed from stage I to stage IV (Table 4 ), with earliest stages being only focal or diffuse wall thickening, to an intermediate stage of focal mass and advanced stage of large mass replacing the entire gallbladder.

As mentioned above, carcinoma GB has three major patterns of presentation on cross sectional imaging. Most common presentation is of a sub-hepatic mass replacing or obscuring the gall bladder (Fig.1) often with invasion of the adjacent liver[1-3]. This finding was seen in 28 $(56 \%)$ of our cases with half of them in an inoperable stage of the disease, a feature reported in other studies also $[7,8]$. Liver invasion was easily detected on CT during the dual phase post-contrast images.

The propensity of carcinoma GB to invade the liver segments $\mathrm{IVb}$ and $\mathrm{V}$ adjacent to the GB fossa is explained by the anatomical characteristic of the GB wall having a narrow lamina propria and only a single muscle layer [3]. The finding of GB abnormality with involvement of the liver segments IVb and $\mathrm{V}$ was one of the most reliable indicators of neoplastic nature in our study.

Approximately $25 \%$ of carcinomas present as an intraluminal mass in the GB [1] (Fig.2). This finding was seen in $10(20 \%)$ cases in this study. These polypoidal intraluminal masses showed homogenous enhancement on the contrast-enhanced images. Areas of necroses or calcification are usually not seen in polypoidal gallbladder carcinoma [1].

Focal or diffuse wall thickening was appreciated in 12 (24\%) cases in this study (Fig. 3). The wall thickening showed enhancement on contrast administration. GB carcinoma presenting with focal or diffuse mural thickening is usually the least common presentation $[1,2]$ and is often difficult to diagnose as GB wall thickening is seen in wide range of clinical settings such as chronic cholecystitis, adenomyomatosis, inadequate GB distension, hepatitis and low protein states [1,9]. The wall is usually $4-13 \mathrm{~mm}$ in thickness and is often asymmetrically thickened and nodular [2]. The differentiation from chronic cholecystitis is difficult and the presence of a highly enhancing thick inner wall layer is considered more indicative of carcinoma whereas an iso-attenuating thin inner wall layer is indicative of chronic cholecystitis[10]. A hypodense halo representing mural oedema in the thickened GB wall has been described as a helpful sign on $\mathrm{CT}$ of cholecystitis rather than carcinoma [2].

Xantho-granulomatous cholecystitis may mimic the above appearances of a mass in the GB fossa with spread of inflammation into liver, duodenum and colon. The presence of intramural hypoattenuated nodules occupying a large area of the thickened GB wall is considered diagnostic of xantho-granulomatous cholecystitis[11]. Hepato-cellular carcinoma invading the GB fossa may mimic carcinoma of the GB. Visualizing a normal GB on CT scan, even if displaced by a tumour, suggests a hepatic primary rather than a GB carcinoma [2].

$\mathrm{CT}$ is superior for evaluating the morphology of portions of GB wall that are obscured by gallstones or mural calcifications on ultrasound [9]. CT is better than ultrasound for detecting lymphadenopathy, especially for N2 nodal stage [3]. In the present study, lymph nodes around the celiac axis and porta hepatis (N1 stage) were involved in six out of 21 patients, whereas retroperitoneal nodal involvement (N2 stage) was seen in the remaining 15 patients.

The diagnostic accuracy of CT scan in staging of the disease and determining resectability has been reported in various studies. Yoshimitsu et al [7], reported an accuracy of $83-86 \%$ in diagnosing the local extent of carcinoma GB, but reported poor sensitivity for $\mathrm{T} 1$ lesions. Kim et al [8], have reported an overall accuracy of $71 \%$ in staging the T-factor of the TNM staging in their study of 100 consecutive cases, with accuracies varying from $79 \%$ for $\mathrm{T} 1 \& \mathrm{~T} 2,46 \%$ for $\mathrm{T} 3$ and $73 \%$ for T4. The accuracy was lowest for thickened GB wall at $54 \%$ and highest for GB mass at $89 \%$.

The role of helical CT in assessing resectability of carcinoma of the gallbladder cannot be over-emphasised. Kumaran et al [12], in their study of 32 patients have reported $93.3 \%$ accuracy in predicting non-resectability using a set criteria. The criteria applied were patients with distant metastases (liver, peritoneum, distant lymph nodes), extensive contiguous local organ spread (duodenum, pancreas, colon), involvement of the secondary biliary confluence and tumoral invasion of the main portal vein or proper hepatic artery or simultaneous invasion of one side hepatic artery and other side portal vein. In the present study Stage IV patients were considered inoperable which accounted for $58 \%$ of our patients. The inoperable patients included those with obstructive jaundice, extensive local spread to duodenum, pancreas and omentum and with overlap of findings of these two groups. 
Ohtani et al [13], found that the sensitivity of CT in detecting the direct spread to the liver of less than $2 \mathrm{~cm}$, more than $2 \mathrm{~cm}$, the extra hepatic bile duct, gastrointestinal tract and pancreas was 65, 100, 50 and $57 \%$ respectively while the positive predictive values (PPV) were 77, 100, 90, 100\% respectively. The PPV was $100 \%$ and $86 \%$ for detection of liver metastasis and involvement of interaortocaval nodes. They concluded that $\mathrm{CT}$ can help in determining resectability and treatment in advanced stages because of high PPV.

In this study a changing epidemiological pattern in the gender distribution as well as a shift of the mean age at diagnosis into the sixth decade of life was observed. There is a corresponding change in morphologic pattern of the disease with progression in the disease stage from wall thickening to mass lesion replacing entire gallbladder. Focal or diffuse mass lesions in the gallbladder fossa with infiltration of liver and second part of duodenum was the commonest pointer for carcinoma gallbladder. Contrast enhanced helical CT was effective in identifying the criteria for resectability of the tumour and in disease staging.

\section{Conflicts of Interest}

None identified

\section{References}

1. Gore RM, Yaghmani V, Newmark GM, et al. Imaging benign and malignant diseases of the gall bladder. Rad Clinics North America 2002; 40: 1307-23.

2. Haaga JR, Herbener EH. The gallbladder and biliary tract. In: Haaga JR, Lanzieri CF, Gilkeson RC, editors. CT and MR Imaging of the whole body. $4^{\text {h }}$ ed. St Louis: Mosby, 2003;
1357-60.

3. Fong Y, Kemeny N, Lawrence TS. Cancer of the Liver and Biliary tree. In: DeVita VT Jr, Hellman S, Rosenberg SA, editors. Cancer : Principles and Practice of Oncology. $6^{\text {th }}$ ed. Philadelphia: Lippincott, Williams and Wilkins, 2002; 1187202.

4. Shath S, Bedford A, Chopra S. Primary gall bladder cancer : recognition of risk factors and role of prophylactic cholecystectomy. Am J Gastroenterol, 2000; 95:1402-10.

5. Rosai J. Gall bladder and extra-hepatic bile ducts. In: Rosai J, Ackerman LV, editors. Surgical Pathology. $9^{\text {h }}$ ed. St Louis: Mosby, 2004; 1044 - 9.

6. Kumar S, Jain A, Jain S. Gallbladder Carcinoma: Experience of 116 cases. Trop Gastroenterol 2001; 22: 172-3.

7. Yoshimitsu K, Honda H, Shinoraki K, Aibe H. Helical CT of the local spread of carcinoma of the gallbladder: evaluation according to the TNM system in patients who underwent surgical resection. Amer J Roentgen 2002; 179: 423-8.

8. Kim BS, Ha HK, Lee IJ, Kim JH. Accuracy of CT in staging of Gallbladder carcinoma. Acta Radiol 2002; 43 : 25.

9. Levy AD, Murakata LA, Rohrmawn CA. Gall bladder carcinoma: radiologic - pathologic correlation. Radiographics 2001;21:295-314.

10. Yun FJ, Cho SG, Park S, Park SW. Gallbladder cacinoma and chronic cholecystitis: differentiation with two-phase spiral CT. Abdom Imaging 2004; 29: 102-8.

11. Chun KA, Ha HK, Yu ES, Shinn KS. Xantho-granulomatous cholecystitis : CT features with emphasis on differentiation from gallbladder carcinoma. Radiology 1997; 203 : 93-7.

12. Kumaran V, Gulati S, Paul B, Pande K. The role of dual phase helical CT in assessing resectability of carcinoma of the gallbladder. Europ Radiol 2002; 12: 1993-9.

13. Ohtani T, Shirai Y, Tsukada K, et al. Spread of gall bladder carcinoma: CT evaluation with pathologic correlation. Abdom Imaging 1996;21:195-201.

\section{ATTENTION CME ORGANISERS}

All service hospitals/institutions organising CME will contribute to MJAFI fund as per the rates given below :-
(a) CME with less than 100 delegates
- $\quad$ Rs. $2500 /-$
(b) CME upto 300 delegates
- $\quad$ Rs. $5000 /-$
(c) CME with more than 300 delegates
- $\quad$ Rs. $10000 /-$ 